\title{
DERECHOS LABORALES EN CHILE: ¿VERDADERA IGUALDAD DE GÉNERO?
}

\author{
Carmina Vásquez Mejías* \\ Universidad de Chile, Chile \\ carmina.vasquez@gmail.com
}

"Todos los hombres nacen iguales, pero es la última vez que lo son"

(Abraham Lincoln)

\begin{abstract}
RESUMEN: Nuestro sistema jurídico, bajo la perspectiva de género, sería descrito como desigual. Esta desigualdad presente en los derechos laborales es una manifestación más de las diferencias por género que la ley establece, sin embargo, en el presente trabajo la tesis no es la tradicional: esta vez, el rol del hombre es el que está limitado en sus relaciones familiares. En términos laborales, la mujer en tanto madre tiene los mayores privilegios legales en relación con los hijos e hijas, los roles económicos que desarrollan en la sociedad, las garantías sociales y las instituciones que sirven de defensa. La esencialización de los roles "mujer-madre" y "hombre-proveedor", en definitiva, no da espacio a una concepción equivalente a los roles de "padre" y "madre", derivando en la desigualdad general entre hombres y mujeres.
\end{abstract}

Palabras clave: paternidad, derechos laborales, desigualdad de género, masculinidad.

ABSTRACT: Our legal system, under a gender perspective is meant to be described as unequal. This inequality is embodied in labor rights as one more manifestation of gender difference established by law, however, in this work our thesis won't be the traditional one: this time, men role is the one limited by familiar relations. In labor terms, women as mother has more legal privileges toward sons and daughters, with economic roles which are developed in society, with social garanties and defense institutions. The essentializing process of "woman-mother" role and "man-provider" gives no space for an equal conception of father and mother roles, becoming general inequality among men and women.

Keywords: fatherhood, labor rights, gender inequality, masculinity.

* Estudiante de Licenciatura en Ciencias Jurídicas y Sociales, sexto semestre. Ayudante ad honorem de la cátedra de Filosofía Moral. 
En la presente investigación reflexionaremos acerca del problema que existe en el derecho laboral respecto a las diferencias entre hombres y mujeres, su evolución histórica y las consecuencias que ésta provoca, pero no desde un punto de vista tradicional sobre la desigualdad sexual, sino que revisando esta vez el menosprecio que se da en nuestro ordenamiento jurídico de "lo masculino" y las posibilidades de ser "padre". Hombres y mujeres aún no logran la igualdad laboral, debido a ciertas medidas paternalistas que subsisten en nuestro ordenamiento jurídico. Con el fin de suscitar una reflexión sobre este importante aspecto, expondremos distintas manifestaciones jurídicas en las cuales la mujer es quien logra los beneficios producto de su condición de "madre" en detrimento de los derechos paternos.

Nuestro Código Laboral señala de forma clara que no existen distinciones entre hombres y mujeres en esta materia; ello en concordancia con la constitución y los tratados internacionales a los cuales estamos suscritos y que, por lo tanto, debemos respetar. Sin embargo, esto no se cumple debido a las múltiples leyes que son aprobadas cada día en beneficio de las mujeres y que dejan en pie desigual a los hombres. En Chile no existe una real preocupación al respecto, especialmente, porque son ellos mismos quienes sienten que es deber del estado asignar al sujeto femenino un rol más bien "doméstico" en la sociedad y en la cual ellas posean ciertas regalías que ellos no tienen. Esto se ve reflejado, por ejemplo, en las facilidades otorgadas para el cuidado de los hijos, puesto que, aunque ambos son procreadores aún no alcanzan los mismos derechos en este aspecto, lo que conlleva considerar el rol femenino como preponderante por sobre el del padre.

Esto es un grave problema social si pensamos el papel que juega, como núcleo fundamental, la familia en nuestra sociedad; definido así, incluso, en el código civil. Si no logramos terminar con estas diferencias a nivel laboral, se seguirá perpetuando el rol patriarcal de nuestras familias, dejando al hombre en una total desprotección de sus derechos de padre. El sujeto masculino mantendrá su estatus de proveedor, mientras la mujer seguirá enmarcada en el ámbito doméstico y desempeñando sólo un rol de madre. Hombre-proveedor, mujer-madre, estereotipos que finalmente perjudican a los hijos, aunque con ellos, se les pretenda proteger.

Los padres actuales, mucho más comprometidos con la educación de sus hijos, se han visto relegados al papel de trabajadores, descuidando sus labores como padres. Sus madres, también trabajadoras, se llevan todo el peso de lo que implica su crianza, puesto que, nuestra actual legislación aún se basa en prejuicios que presentan a la mujer (como históricamente se ha hecho) en su rol de madre. Junto a ello, se efectúan políticas de protección hacia ella, lo que demuestra nuestro paternalismo no sólo respecto al resguardo de la mujer embarazada y la familia, sino también, en materia de jubilación, en el que, de igual forma, se ve perjudicado el hombre, tal como veremos a continuación.

La situación actual en Chile ha ido cambiando a lo largo de nuestra historia y ya no corresponde ver a la mujer como el sexo débil. La excesiva protección hacia sus derechos, por ser considerada aún como "dueña de casa" y no como trabajadora en igualdad de condiciones, ha 
hecho que sea el sujeto masculino quién sufra las consecuencias. Es él quien mantuvo su posición de hombre-proveedor mientras ella es la que decide hoy ser mujer-trabajadora, antes que mujer-madre. El hombre, en cambio, no tiene permitido tomar estas decisiones: su rol en nuestra sociedad está delimitado. Es por esta evolución social que las mujeres ya no son sujetos con incapacidad relativa, del mismo modo en que ellos se han ido comprometiendo con el cuidado de sus hijos, transformándose en padres "activos". Ante este nuevo panorama se hace imprescindible que nuestras políticas laborales cambien y se renueven hacia una verdadera igualdad en materia de género.

Sobre estas bases se plantea la siguiente interrogante: ¿Qué características debería poseer nuestra legislación laboral para tender a una real paridad de género?, esta pregunta constituye la estructura de nuestra investigación y nos remite al propósito de entender las verdaderas desigualdades en materia laboral: por qué se producen, en qué medida son necesarias realmente y, de esta forma, conseguir dar una respuesta satisfactoria a este problema.

Para cumplir con este propósito los objetivos específicos del informe serán determinar las políticas laborales existentes en nuestra legislación, logrando distinguir las ventajas y desventajas que ellas conllevan; analizar sobre qué inspiraciones (jurídicas, religiosas, económicas, etc.) se basan las normas actuales de distinción de género en materia laboral y determinar qué leyes deben ser mantenidas por nuestro ordenamiento jurídico y cuáles debieran ser eliminadas para lograr la verdadera igualdad en dicho asunto.

Con el fin de identificar los elementos que componen las leyes laborales actuales, esta investigación tendrá un carácter exploratorio, puesto que se indagará en diversas fuentes que tratan tanto el tema de los derechos laborales como las distinciones de género. A la par, poseerá un carácter descriptivo, ya que se caracterizarán las leyes laborales que se debieran implementar en nuestro país para producir una real paridad entre hombres y mujeres, acorde con nuestra sociedad. La clase de material que se utilizará será de tipo documental (libros, revistas) y empírico, en cuanto se utilizan datos producidos por encuestas sobre las diferencias laborales existentes.

Una investigación vinculada a las diferencias laborales es importante desde tres ejes: el metodológico, el teórico y el práctico. Desde lo metodológico, la relevancia radica en la poca bibliografía que existe en torno al tratamiento conjunto de dos áreas, la igualdad de género tendiente a favorecer al sexo femenino y las leyes laborales, por lo que este trabajo suple en cierta forma la falencia, alentando al desarrollo multidisciplinario sobre el tema. En lo teórico, el valor de la investigación en el ámbito jurídico y de género acerca de las leyes laborales, reside en su reciente incorporación en debates políticos, sobre todo en la nueva ley de maternidad en la que se aísla el rol de padre, junto a la escasa legislación que existe en Chile al respecto, en contraste con la mayoría de los países del mundo. Mientras que en lo práctico, se pretende determinar 
qué características debiese tener una nueva legislación laboral en relación al estudio de género y las políticas destinadas hacia dicha igualdad, debido a que no existen motivos fundados para que se hagan estas distinciones contrarias al mismo derecho.

\section{DERECHOS LABORALES Y SU EVOLUCIÓN HISTÓRICA}

\section{I DERECHOS LABORALES A LO LARGO DE LA HiSTORIA}

Los derechos laborales chilenos nacen el 4 de junio de 1559 con la promulgación de las ordenanzas: "Para el buen gobierno, pacificación y defensa de Chile" de Fernando de Santillán", dictadas en el marco de las aspiraciones religiosas y morales que desde España habían establecido los reyes católicos en las capitulaciones de santa $\mathrm{fe}^{2}$. En éstas, se imponen una serie de normativas sobre descanso, vacaciones, jornadas de trabajo y todo lo referente a la regulación laboral en Chile, sin embargo, ellas no se cumplían. Debido al alto nivel de analfabetismo en nuestro país era difícil que los empleados entendieran sus derechos escritos en las ordenanzas, por lo que además de confusos y muchas veces contradictorios, eran ineficaces.

En el siglo XIX esto comienza a cambiar, de forma paulatina, producto del proceso codificador de esos años, en el cual se aprecian las primeras normas laborales chilenas, tales como, el Párrafo VII y el XXVI del código civil sobre criados domésticos y a los que le siguieron el privilegio de pobreza, la jubilación de empleados civiles (1857), reglamento de matrícula de gente de mar (1898), etc. Un estudio revela que a principios de 1900 ya se gestaban nuevas ideas que pronto se convertirían en "la cuestión social"3. La migración campo-ciudad estaba haciendo estragos en las grandes metrópolis, debido a la sobrepoblación y la falta de previsión sobre este problema, las urbes estaban colapsadas y se creaban círculos de pobreza alrededor de ellas lo que, más adelante, se conocerían como "poblaciones callampas". Esta situación provocó el despertar de una clase nueva para esos tiempos, la clase obrera, a la vez que en Santiago, producto de las condiciones de hacinamiento en que se vivía, las ideas anarquistas se hacían más potentes. En 1910, alrededor de 100.000 personas se hacinaban en 25.000 conventillos. La iglesia católica intentó canalizar estas nuevas ideas en la encíclica Rerum novarum, que buscaba el equilibro entre los trabajadores y los empleadores, para evitar la sobreexplotación de los empleados "Lo común debe administrarse con toda integridad, de modo que la cuantía del socorro esté determinada por la necesidad de cada uno; que los derechos y deberes de los patronos se conjuguen

1 De Santillán, Fernando. Declaración de lo que el Licenciado Fernando de Santillán, oidor de la Audiencia de Lima, proveyó para el buen gobierno, pacificación y defensa de Chile, 4 de junio de 1559. En: Jana, Álvaro y Pinto, Sonia, Fuentes para la Historia del trabajo en Chile. Santiago, Chile: Andrés Bello, 1982, p. 19.

2 Bravo Lira, Bernardino. Derecho común y derecho propio en el Nuevo Mundo. Santiago, Chile: Editorial Jurídica, 1989, p. 14.

3 Thayer Arteaga, William. Notas sobre génesis y desarrollo del derecho del trabajo en la República de Chile. En: TApia, Francisco (comp.). Derecho del Trabajo: Normas y realidad. Obra colectiva de homenaje al profesor don Ramón Luco. Santiago, Chile: Universidad Andrés Bello, 1993. 
armónicamente con los derechos y deberes de los obreros" ${ }^{4}$. Ello, sobre la base de la religión católica, la cual promulga que todos los hombres somos iguales en dignidad y por lo tanto empleadores y trabajadores deben cumplir sus deberes, respetándose mutuamente.

Chile se incorpora a un organismo internacional de prestigio, la Organización Internacional del Trabajo (OIT), por la Ley No 3.553 en 1919. De ello nacen las principales ideas sobre la dignificación del trabajo que encontramos hoy. Éstas fueron incorporadas en nuestro país mediante la promulgación de las leyes sociales que hizo el presidente Alessandri Palma, culminando en el Código del Trabajo remitido el 8 de junio de 1921 al Congreso y que se cristalizaron en 1924 producto de la presión de los militares. Fueron decretadas con algunas enmiendas por Carlos Ibáńez del Campo, por el Decreto Fuerza Ley 178, en mayo de 1931. En esta legislación primaba el poder intelectual sobre el físico, dando mayores beneficios al primero de estos grupos. En 1959 Chile hace las primeras ocho ratificaciones de América del Sur, debido a la gira de Albert Thomas quien intentaba que los países latinoamericanos participaran y ratificaran los tratados suscritos. ${ }^{5}$

En 1987 se establece el segundo código a cargo del General Augusto Pinochet. La idea de país que había nacido de Carlos Ibánez estaba en crisis para 1974, por lo que los organismos de previsión se unieron en la Instituto de Previsión Social ${ }^{6}$. En este nuevo código el estado se enmarcó en una política subsidiaria, regulando la duración de la jornada laboral, lugar de trabajo y remuneración de horas extras, entre otros. En 1989 el texto se refundió y, aunque se han ido incorporando nuevos preceptos, no ha cambiado esencialmente.

\section{i.2 Distinciones E igualdades entre los DeRechos laborales Del Hombre y de la MUJER}

Chile ha logrado de forma gradual la incorporación de la mujer en el mundo laboral. Las estadísticas nos indican que para el año 1920 solo el 19,2\% trabajaban y esto ya entrado el S. $\mathrm{XX}^{7}$, sin embargo, el rol del sujeto femenino como "mujer-madre", no ha terminado ni siquiera hoy, las políticas públicas han ampliado el rol de la mujer trabajadora a la par con el aumento de sus beneficios maternales.

El primer paso que se dio para lograr la participación laboral femenina fue la educación. En 1898 se fundó la primera escuela de niñas y a mediados de siglo ya existían 139 con una

4 Rerum Novarum. Sobre la situación de los obreros. <En línea>. [Citado 9 de Octubre de 2010]. Disponible en la World Wide Web: <http://www.vatican.va/holy_father/leo_xiii/encyclicals/documents/hf_l-xiii_enc_15051891_rerum-novarum_sp.html>

5 Conmemoración 90 años de la OIT. América en la OIT. <En línea>. [Citado 8 de octubre de 2010]. Disponible en la World Wide Web: <http://90.oit.org.pe/?p=7>

6 Historia de la INP. Integración ex cajas particulares. <En línea>. [Citado 8 de octubre de 2010]. Disponible en la World Wide Web: < http://portal.ips.gob.cl/portal/inp/datos_historia.htm>

7 Bravo, David. Trabajo: dignidad y cambios. El mercado laboral chileno. En: Tironi, Eugenio et al. Cuánto y cómo cambiamos los chilenos. Balance de una década 1992-2002. Santiago, Chile: Instituto Nacional de Estadísticas, 2003, p.136. 
matrícula total de 6.400 alumnas. En 1877 Miguel Luis Amunátegui, ministro de justicia, permite el ingreso de las mujeres a las universidades. Eloísa Díaz y Ernestina Pérez fueron las primeras doctoras al titularse en 1887 y Matilde Throup, en el ańo 1892, se convierte en la primera abogada, en 1907 existían 10 mujeres profesionales, 3 abogadas y 7 médicos. $^{8}$

En el año 1925 el gobierno dicta el decreto Ley No 328, importantísimo dentro del análisis de género de los derechos laborales, ya que crea "el patrimonio de la mujer casada", esto significa que la mujer debía recibir directamente su remuneración por los trabajos realizados, puesto que antes de ésta, el salario era entregado a su cónyuge. En 1934 esta ley fue perfeccionada y se aceptó que todos los bienes que la mujer obtuviese podían ser administrados por ella, sin intervención del hombre. Al crearse la Ley No 7.612, se acepta la posibilidad de casarse en régimen conyugal de separación de bienes. La ley 10.271 de 1952 otorga mayores derechos sobre los bienes a la mujer separada, lo cual se acentúa en 1986.

El camino de las mujeres para abrirse un lugar entre los hombres no fue fácil, sólo en 1935 logran el voto femenino para las elecciones municipales y en 1949 se les concede para las parlamentarias y las presidenciales bajo el gobierno de Gabriel González Videla. En 1951 es electa la primera mujer diputada, Inés Enríquez, representante de la provincia de Concepción. Posteriormente, llega a ser presidenta de la cámara de diputados. ${ }^{9}$

Las mujeres, producto de la cuestión social lograron algunos derechos laborales que se fueron ampliando a lo largo de la historia, al igual que la de los hombres; sin embargo, mientras éstos ganaban terreno respecto a su rol de trabajadores, el sujeto femenino obtuvo, además, beneficios como madre, doblando así los derechos masculinos.

\section{SITUACIÓN ACTUAL DE LOS DERECHOS LABORALES. PUNTOS CRÍTICOS}

\section{I Situación ACtUAL RESPECTO A LOS DERECHOS LABORALES}

Aunque en nuestro Código del Trabajo se exprese que no existen diferencias por género, comprobamos en la práctica que esto no es así, ya que la mayoría de las distinciones se hacen precisamente por ello. La persistencia de estas medidas en el Código del Trabajo no se entienden en realidad y sus explicaciones son vagas. Lo único que se logra con éstas, como ya hemos mencionado, es conducir a la desigualdad los derechos del hombre-padre, a la vez que el sujeto

8 SÁnchez, Karim. "El ingreso de la mujer chilena a la universidad y los cambios en la costumbre por medio de la ley 1872 1877”, en: Historia No 39, Vol. 2, Santiago, Chile, julio-diciembre 2006. Instituto de historia. Pontificia Universidad Católica de Chile, pp. 497-529.

9 Pardo, Adolfo. Historia de la mujer en Chile. La conquista de los derechos politicos en el S.XX. <En linea>. [Citado 6 Octubre 2010]. Disponible en la World Wide Web: <http://www.critica.cl/html/pardo_01.html> 
femenino no pueda descuadrarse de su papel de mujer-madre y, en consecuencia, se perpetúe la discriminación. Esto se refleja en el hecho de que en Chile las mujeres ganan un 38,6\% menos que los hombres en iguales condiciones laborales ${ }^{10}$.

A continuación expondremos las principales instituciones que se mantienen en nuestro derecho y que refuerzan la desigualdad en torno al sujeto masculino:

- Fuero maternal: "Consiste en la imposibilidad, por un periodo determinado, de ser despedida de su trabajo, o que el empleador(a) ponga término a la relación laboral sin una autorización judicial previa. La duración del fuero comprende desde el momento de la concepción, es decir, desde el inicio del embarazo, hasta un año después de expirado el periodo postnatal o el periodo postnatal suplementario. No hay trabajadoras excluidas de este beneficio". ${ }^{11}$

Podemos ver que en este beneficio queda absolutamente excluido el padre. Con él se pretende proteger los derechos del que está por nacer sin considerar que esto no siempre es lo que se logra. Como expliqué anteriormente, los padres, muchas veces, son los que tienen un sueldo superior y por lo mismo, al pensar en el bien del que está por nacer, es el hombre quién debería tener este privilegio. Además de esto, el fuero propicia la no contratación del sujeto femenino en el mercado laboral, ya que, por el hecho de ser mujeres, el empleador no puede despedirlas independiente de la eficiencia con que realicen su trabajo. Por lo tanto tenemos dos fenómenos, el primero es que las mujeres no son contratadas porque no pueden ser despedidas, lo que coarta la libertad del empleador y, por otra parte, no favorece realmente al que está por nacer, puesto que se otorga en razón del género sin considerar la situación económica de los cónyuges.

- El prenatal: va desde las 6 semanas anteriores al parto y postnatal hasta 12 semanas después del parto. Esta institución es la que demuestra con mayor esplendor las desigualdades en materia laboral y una de las más nefastas para todo padre, ya que se lo excluye totalmente de su rol con el fin de enmarcarlo en el estereotipo de hombre proveedor. Esto conlleva la sujeción del sujeto femenino al papel de madre, sin que se le permita desarrollarse como trabajadora. Es lógico que la mujer tenga un tiempo para descansar antes del parto y después de éste, sin embargo, no todas requieren el mismo tiempo, de la misma manera en que el cuidado del recién nacido puede ser adoptado tanto por el padre como por la madre, un hermano o abuelo, según quién sea el mejor capacitado para desempeñar dicha función.

La figura del padre está, en este sentido, completamente desdibujada, puesto que sólo tiene 4 días para estar con su hijo, una gran diferencia si pensamos que la mujer tiene 3 meses y que actualmente se discute en la cámara que se prolongue a 6. Muchas madres sufren depresión

10 Trabajo y equidad. Encuesta CASEN Diferencias salariales. <En línea>. [Citado 6 de octubre de 2010]. Disponible en la World Wide Web: <http://www.trabajoyequidad.cl/view/viewArticulos.asp?idArticulo=30>

11 Dirección del trabajo. Derechos de la mujer trabajadora. <En línea>. [Citado 6 Julio 2010]. Disponible en la World Wide Web: <http://www.dt.gob.cl/legislacion/1611/w3-article-62965.html> 
post parto, por lo cual no se encuentran capacitadas para responsabilizarse por el bienestar de sus hijos. El estado, por tanto, no puede obligarla a asumir este rol, ya que, más que generar un beneficio para el recién nacido puede resultar perjudicial para éste.

- Permiso por hijo menor de 18 años afectado por una enfermedad terminal en su fase final, o gravemente enfermo con probable riesgo de muerte: La madre trabajadora tiene derecho a un permiso para ausentarse de su trabajo por el número de horas equivalentes a 10 jornadas ordinarias de trabajo al año, distribuidas a elección de ella (Art. 199 bis código del trabajo). Los derechos como padre son absolutamente violados por esta ley, puesto que se le ve como simple proveedor. Se le niega el derecho a estar con su hijo antes de morir.

- Derecho de cuidado al niño menor de un año que sufra enfermedad grave: Dispuesto en el artículo 199 del Código del Trabajo, cuando la salud de un niño menor de un año requiera la atención en el hogar con motivo de enfermedad grave, la madre trabajadora tiene derecho a un permiso y subsidio por el período que el respectivo servicio determine. Es del caso señalar que si ambos padres son trabajadores, cualquiera de ellos y a elección de la madre, puede gozar del permiso y del subsidio en cuestión. Es del caso señalar que la norma legal establece que el padre gozará de los beneficios cuando la madre hubiere fallecido o él tuviere la tuición del menor por sentencia judicial.

Es claro aquí que la visión de nuestra sociedad es absolutamente anacrónica. El rol de padre está subordinado a los deseos y necesidades de la madre, sin poseer una libre elección de paternidad. Sólo en el caso de que la mujer no cumpla con su función tendremos a un padre de forma subsidiaria.

- Derecho a sala cuna: Es obligación del empleador que cuente con 20 o más trabajadoras, mantener una sala cuna anexa e independiente del local de trabajo donde las mujeres puedan dejar a sus hijos menores de dos ańos y alimentarlos.

Un empleador puede cumplir con esta obligación de 3 maneras, a saber: manteniendo en forma individual una sala cuna, manteniendo en forma conjunta una sala cuna con otros empleadores, pagando a una sala cuna los gastos que signifique el costo del beneficio donde la mujer lleve a su hijo o hija (s).

El sujeto masculino nuevamente es privado de su rol de padre. Si la mujer trabaja parttime o tiene su propio negocio podría ser difícil para ella hacerse responsable plenamente de los niños, sin embargo, el padre no puede acceder a salas cunas por el simple hecho de ser hombre. Esto es abiertamente una discriminación de género. En este caso no quedaría más que pagarle a una empresa privada para que cuide a los menores, solución que no puede ser satisfecha por muchas familias que no poseen el dinero suficiente para gozar de estos privilegios. En ese sentido, la discriminación por parte de esta ley sería doble: en primer lugar por género y en segundo, por la posición socioeconómica de la familia. 
- Alimentación del hijo o hija: En virtud de la nueva Ley 20.166, las mujeres podrán hacer uso de este derecho, por el lapso de una hora, sea que los menores estén en sala cuna, en su hogar o donde la trabajadora lo elija. La madre podrá disponer de esa porción de tiempo en alguna de las siguientes formas a acordar con el empleador(a): a) en cualquier momento dentro de la jornada de trabajo; b) dividiendo la hora de que dispone en dos porciones, c) o postergando o adelantando el inicio y/o término de la jornada. En el caso de las empresas que tienen sala cuna, el tiempo empleado en traslado se considerará trabajado y el coste del transporte es por cuenta del empleador(a).

Las mujeres y hombres nacen iguales en capacidad y derecho, por lo tanto, los hombres sí son capaces (y responsables) de la tarea de alimentar a sus propios hijos. No se entiende por qué este beneficio es exclusivo de la mujer si, en general, ni siquiera se respeta esa hora, por el contrario, se utiliza para entrar una hora después o salir una hora antes del trabajo. Si ambos contaran con el mismo derecho propiciaría que los padres pasaran más tiempo con su familia. Por supuesto, no podemos obviar el hecho de que naturalmente es la mujer quién, durante los primeros meses, puede alimentar al hijo, pero este beneficio se aceptaría sólo si efectivamente esa hora se utilizara para ello.

Estas instituciones nacen principalmente para proteger al hijo o hija que está por nacer, darle seguridad y hacerlo crecer en un ambiente adecuado para desarrollarse como persona. Como lo dice el Código Civil "la familia es el núcleo fundamental de la sociedad. El matrimonio es la base principal de la familia" 12 , sin embargo nuestras leyes laborales no reconocen este principio sino, más bien, asumen que las mujeres con sus hijos son quienes constituyen este núcleo. Todas las leyes tendientes a "proteger a la familia" se refieren a la relación que mantienen las madres con sus hijos, desdibujando el rol de padre. La alimentación del menor, el derecho a sala cuna, el cuidado al hijo enfermo, el fuero maternal, son leyes que protegen a la mujer debido a la creencia de que con ellas se está protegiendo al niño, sin embargo, el papel que debiera cumplir el padre en la crianza, alimentación y cuidado de éste no se considera más que como proveedor. El padre, así, debe pagar pensión alimenticia al hijo hasta que éste cumpla los 21 años o los 28 si es que éste se encuentra estudiando ${ }^{13}$, no obstante, se lo absuelve de sus responsabilidades afectivas.

- Jubilación: en Chile la jubilación es de 65 años los hombres y 60 años las mujeres. Las mujeres estadísticamente viven más que los hombres: la expectativa de vida en el hombre es de 73 años, mientras que el de las mujeres es de $79^{14}$, por lo que no resulta comprensible que sean éstas las que jubilen antes; siguiendo esta lógica, los hombres, al vivir menos, deberían hacerlo

12 Ley 19.947, ley de matrimonio Civil, Art.1, inc.1.

13 Ley 14.908, Sobre abandono de familia y pago de pensiones alimenticias. Y sus modificaciones y artículos del 321 al 337 del Código Civil.

14 Expectativa de vida <En línea>. [Citado 6 Octubre 2010]. Disponible en la World Wide Web: <http://web.latercera.cl/medio/ articulo/0,0,3255_5726_35710797,00.html> 
primero. Este hecho lleva a que el sujeto femenino gane un $10 \%$ menos que el masculino al momento de jubilar a su edad legal. También las mujeres cotizan después que los hombres, tienen lagunas previsionales por la maternidad, ganan menos, se jubilan antes y viven más. Todo lo contrario pasa con los hombres quienes, a pesar de todo esto, no pueden realmente disfrutar de ésta ya que se retiran de su empleo con muy poca esperanza de vida: el $60 \%{ }^{15}$ de los trabajadores se jubila de forma anticipada. Esta situación ha suscitado varias propuestas al respecto sin que se hayan concretado, producto de las dificultades que conlleva.

Actualmente en nuestro país el Código del Trabajo, artículo 2, define discriminación como: "Son las distinciones, exclusiones o preferencias basadas en motivos de raza, color, sexo, edad, estado civil, sindicación, religión, opinión politica, nacionalidad, ascendencia nacional u origen social, que tengan por objeto anular o alterar la igualdad de oportunidades o de trato en el empleo y la ocupación”. En éste, además, se señala explícitamente que no deben existir medidas discriminatorias, tomando el sexo como una de ellas. Sin embargo en cada una de estas leyes existiría este tipo de discriminación, puesto que, no se consideran relevantes las capacidades de cada uno en la concesión de beneficios, tampoco factores tales como, la idoneidad para el cuidado, la cercanía del trabajo, expectativa de vida, etc.; por el contrario, el factor género (y sus respectivos estereotipos) resulta determinante a la hora de adjudicar ciertos derechos.

\subsection{Consecuencia de Desigualdad Que conlleVan estos Derechos laborales}

Lo que conlleva esta desigualdad laboral es fácil de imaginar según las premisas anteriores. La primera consiste en las diferencias salariales entre hombres y mujeres: éstas reciben sólo un $61,4 \%$ de lo que ganan los hombres, esto sucede porque para los empleadores es mucho más caro contratar mujeres. Según datos cuantitativos la contratación estaría en un $31 \%{ }^{16}$ más caro producto de los costos "no salariales" que representan, dentro de los que se consideran todos los beneficios maternales que tienen y de los cuales ellos no pueden gozar. Esto lleva una doble complicación: primero, para los empresarios, puesto que es en un costo innecesario contratar mujeres si deben hacerse cargo de la familia de éstas, la segunda es para ellas, puesto que, discriminadas de los trabajos a los que podrían acceder, aceptan pagos menores como algo natural. Junto a esto, se ven obligada a cumplir un rol de madre paralelo al de empleadas.

El sujeto masculino, por su parte, y a lo que nos avocamos en este trabajo, no tiene una igualdad con respecto a los derechos laborales relacionados a la familia, incluso se podría decir que no pertenece a ella. Se desdibuja la figura paterna para terminar siendo, simplemente, el hombre-proveedor, no el hombre-padre que buscamos según la definición de familia expuesta

15 Expectativa de vida <En línea>. [Citado 6 de septiembre de 2010]. Disponible en la World Wide Web: <http://web.latercera. cl/medio/articulo/0,0,3255_5726_35710797,00.html>

16 Todaro, Rosalba y Lerda, Sandra. Estudio de Costos Laborales por Sexo. En: Informe Final de Consultoría al Servicio Nacional de la Mujer-SERNAM, Santiago, Chile, 1996. 
en la ley de matrimonio civil, base de la sociedad. Un núcleo sin padre es lo que en la actualidad estamos creando por medio de estas leyes laborales, en las que el hombre no es igual a la mujer y no tiene el mismo derecho que ella de ver crecer y estar con sus hijos, a la vez que descuidan sus obligaciones, en este sentido, porque la ley se los impide, no porque no lo deseen.

Con respecto a la jubilación, caso distinto al de las familias, podemos ver que esta medida, aunque no tiene ninguna razón de ser, presenta múltiples consecuencias. En primer lugar, las mujeres reciben menos dinero al jubilarse por el simple hecho de hacerlo cinco años antes. Esto no se comprende si consideramos que ellas viven más. Por ejemplo, muchas trabajan 20 años y viven 27 más al momento de retirarse: "Las mujeres afiliadas declaran haber estado inactivas en promedio $35 \%$ de su vida laboral y sus sueldos son $19 \%$ más bajos que los de ellos" 17. Además de esto, el mismo estudio señala que el $60 \%$ de los trabajadores opta por la jubilación anticipada, lo que demuestra que tanto hombres como mujeres se retiran de forma anticipada por razones distintas: ellas no tienen mucho incentivo para trabajar, debido a los bajos sueldos que perciben; ellos, en cambio, al vivir menos buscan disfrutar sus últimos años sin necesidad de trabajar.

"Mujer y trabajo: el 30,4\% de las mujeres declaró trabajar fuera del hogar, mientras que un 64,1\% se declaró como dueña de casa: el 90,1\% declaró tener hijos. De las que no trabajan, el 58,3\% declaró que 'no trabaja porque no desea': el 10\% de estas dijo que hacerlo 'es malo para la mujer y la familia', mientras que el 37,8\% dijo que 'es bueno para la mujer, pero malo para la familia'. El 53,6\% de estas indica que es malo porque 'se opaca al marido". ${ }^{18}$

En esta encuesta se refleja la idiosincrasia chilena, en la que la mujer tiene opciones, mientras el hombre se encuentra enmarcado en el estereotipo de hombre-proveedor. La mujer tiene la posibilidad de optar por el trabajo o por el rol de madre, sin embargo, el hombre no goza de esta elección, no puede decidir no trabajar y quedarse a cuidar a sus hijos. La mujer no trabaja porque no quiere trabajar, el hombre tiene que trabajar, sin importar sus deseos. Estas leyes laborales permiten que se mantenga la estructura del hombre coartado en su rol paterno.

\section{ALTERNATIVAS PARA EVITAR LA DESIGUALDAD EN LOS DERECHOS LABORALES}

\section{I CAmbios jurídicos En El Derecho laboral}

Fuero maternal: Se debe crear mentalidad en los individuos de nuestra sociedad para que se logre una verdadera igualdad, por lo tanto, debido a que el fuero maternal se creó espe-

17 LARRAÍn, Guillermo. Estudio SAFP. Jubilación legal y anticipada.

18 VAldÉs, Ximena et al. Puertas adentro. Masculino y femenino en la familia contemporánea. Santiago, Chile: LOM, 2006, p. 72. 
cialmente para "proteger al que está por nacer", esta institución no cumple en realidad con la función para la que se creó. Muchas veces no es la mujer la que gana más que el hombre y, en concordancia con lo señalado, éstas son más caras para los empleadores, por lo que resulta lógico que el contratante quiera despedirla al momento en que quede embarazada por los costos que implica. Si cambiáramos el sistema jurídico sobre la base de la igualdad real, el empleador no tendría que asumir mayores costos por contratar hombres o mujeres.

Al ser pensada, esta medida, como protección al que está por nacer, el fuero debiera aplicarse para el padre o la madre indistintamente, según lo decida la pareja y, de este modo, escoger según factores tales como: el que está en mayor riesgo de ser despedido por hacerse cargo del hijo o quien gane más y que, por ello, sea más favorable que conserve su trabajo. Si la pareja decidiera a quién entregarle el fuero según los beneficios familiares particulares, podríamos llegar a una igualdad real; los empleadores no tendrían prejuicios antes de contratar y el que está por nacer podría realmente tener seguridad económica, asimismo, el empleador no estaría amarrado a mantener a mujeres ineficientes en su trabajo.

Pre y post natal: Nuestro país ha ido evolucionando lentamente con respecto a los derechos laborales del resto del mundo, en países europeos, como España, el postnatal es compartido. Las leyes le dan 18 semanas para la pareja lo que implica que ellos pueden elegir quién se toma ese tiempo: puede ser la mujer, el hombre o 9 semanas cada uno. Actualmente se discute que este derecho se haga obligatorio para cada uno de los padres, 9 semanas para la madre y 9 para el padre, lo que lo convertiría en un deber de cuidado, protección y relación con el hijo. Esta medida sí nos llevaría a una verdadera igualdad en los derechos laborales, ya que no se discriminaría arbitrariamente los roles que cada uno debe cumplir en la sociedad. Ambos podrían elegir cuánto tomar cada uno, en forma conjunta, sin ser la sociedad quien decida por ellos. Actualmente, esta ley es abiertamente discriminatoria y sólo lleva a que, debido a las contribuciones que debe pagar la empresa a la mujer embarazada, no se las contrate y se prefieran a los hombres, sin contar con que para encontrar un reemplazo y capacitarlo por 18 semanas (6 prenatal, 12 postnatal) es inviable.

Tanto el padre como la madre deben estar con sus hijos cuando éstos sufren una enfermedad grave, cuando hay peligro de muerte, en los momentos difíciles. Privarle el derecho de permanecer al lado de sus padres a un menor que está muriéndose es injustificado. En este caso, es fundamental que en los últimos momentos de su vida los padres puedan acompañar a su hijo y el hijo, asimismo, se sienta acompańado por sus padres. Como en los casos anteriores, lo que llevaría a una real paridad sería que la pareja pudiera repartirse este derecho y cumplir su rol de padres sin descuidar su trabajo. Lo que puede significar que gocen de este beneficio a la vez si es necesario para el hijo.

Lo mismo se aplicaría en caso de enfermedad de un niño menor de un año. Al tener ambos los mismos derechos según el Código del Trabajo, cualquiera de ellos, debería poder au- 
sentarse en caso de grave enfermedad para su hijo, teniendo preferencia quien pueda auxiliarlo de forma más oportuna y no decidir según su sexo. Por ejemplo: quién trabaje más cerca del domicilio del menor.

En el caso de las salas cunas es inapropiado pensar que sólo la mujer cuando trabaja puede recurrir a ella. Tanto hombres como mujeres son padres y, por lo tanto, ambos deberían tener derecho al uso de éstas en sus trabajos respectivos; ello evitaría también la discriminación laboral contra la mujer y la radicación de empleadores que contratan hasta 19 mujeres para no tener que implementarlas en los empleos. Si hombres y mujeres reparten sus roles familiares, cualquiera de los dos podría tener a sus hijos en salas cunas anexas a las empresas.

Respecto a la alimentación de sus hijos, tanto hombres como mujeres son capaces de nutrirlos, por lo tanto, ambos deberían poder contar con esa hora para hacerlo según lo que la pareja decida. De esta forma, se le asegura al hijo un padre y una madre presente y no simplemente una mujer-madre y un hombre-proveedor. Si bien, se entiende que son las mujeres las que amamantan, esta hora no resulta efectiva si sólo sirve para que éstas salgan una hora más temprano del trabajo; en ese caso, no cumple con el fin para lo que fue establecido.

\subsection{HaCia UNA VERDADERA IGUALDAD DE DERECHOS}

La sociedad chilena tiene importantes vestigios de paternalismo hacia las mujeres y esto se ve especialmente reflejado en los derechos laborales y sus diferencias discriminatorias de género. Esto redunda en que en Chile se mantenga una desigualdad que actualmente favorece a las mujeres en el ámbito familiar, de la misma forma en que las perjudica en el aspecto económico; asimismo, mientras los hombres son favorecidos en el ámbito económico, en el ámbito familiar son desdibujados como figuras paternas.

Para que nuestra sociedad logre estándares internacionales es necesario que sus leyes sean también de este tipo, por lo tanto, es sumamente importante que se modifiquen las leyes laborales para que consagren la verdadera igualdad entre hombres y mujeres. Con estas medidas simples que reconocen el rol de padre del sujeto masculino y deslindan a la mujer de su estereotipo de madre podremos tener un país más justo y una familia unida.

Lo visto anteriormente es sólo el primer paso hacia una paridad real, puesto que, para cambiar en esencia las leyes laborales es necesario comenzar por cambiar la mentalidad de las personas. Un primer paso ganado por los hombres en esta batalla fue haber logrado ampliar el post-natal masculino de 1 día a 4 y en la actualidad se debate si debe extenderse aún más. Otro gran paso se dio en el 2008 cuando se admitió la posibilidad de que si la madre se ausenta o pierde la tuición del niño sea el hombre quien pueda acceder a los beneficios de la madre en subsidio de ella. 


\section{CONCLUSIÓN}

En nuestro país aún perduran prácticas que van en contra de la igualdad de género que propugnamos y que, incluso, se encuentran consagradas en las leyes. Sin embargo, nuestra sociedad ha ido evolucionando a grado tal que ya parece lógico que los roles se distribuyan de forma igualitaria, lo que implica la necesidad de que las leyes cambien en favor de las personas a quienes sirven. Esta nueva forma de entender la paridad en favor de ambos sexos y no sólo de las mujeres, ha llevado a que se pongan en el tapete temas tan importantes como una mayor participación del hombre en el seno familiar, por medio de la admisión de los derechos del padre, como lo es el post-natal.

Los derechos laborales han sufrido grandes procesos para ser lo que son ahora y conseguir reconocer los derechos fundamentales de los trabajadores, de la misma manera, las mujeres han intentado ganar su espacio en esta sociedad y lograr la igualdad con el sujeto masculino. Sin embargo, al momento de alcanzarla, estos derechos se han desviado, entregándole injustamente mayores regalías a ellas que a los hombres. Las diferencias de género no son únicamente salariales sino que muchas veces, por reconocer los derechos de las mujeres, los del hombre no evolucionan. Esperamos que éstas vayan cambiando a medida que este tipo de controversias salgan a la luz pública y sean cuestionadas por la propia ciudadanía.

Es urgente que se legisle sobre la igualdad de los derechos laborales entre hombres y mujeres, en primer lugar, para dar al hombre el lugar que se merece en ésta. Los niños tienen derecho a ser criados por sus padres y madres, así como los padres deben obtener las mismas regalías que las madres para desempeñar su rol; esto mejorará las relaciones entre padre e hijo, a la vez que logrará terminar con la asociación mujer-madre y padre-proveedor, entendiendo que ambos pueden desempeñar ambos roles indistintamente.

Esta igualdad en los derechos no sólo llevará al hombre a tener una mejor relación con sus hijos, sino que ayudará también a que la mujer no sea excluida por el hecho de ser madre. Si ambos se reparten las cargas y beneficios laborales el empleador no podrá discriminar por quien es más caro al contratar y deberá, por lo tanto, terminar con las preselecciones en las que el sujeto femenino se ve menoscabado.

Por otra parte, es sumamente importante también que hombres y mujeres jubilen a la misma edad o, incluso, sea ella quien lo haga después, debido a sus mayores probabilidades de vida. Ambos, podrían contar con previsiones más dignas que le permitieran mantenerse, de la misma forma, en que podría reducirse la edad de jubilación para los hombres en beneficio de una vida más tranquila en su último período. 


\section{REFERENCIAS BIBLIOGRÁFICAS}

Bravo, David. “Trabajo: dignidad y cambios. El mercado laboral chileno”. En Tironi et al. Cuánto y cómo cambiamos los chilenos. Balance de una década. Santiago, Chile: INE, 2003.

Bravo Lira, Bernardino. Derecho común y derecho propio en el Nuevo Mundo. Santiago, Chile: Editorial Jurídica, 1989.

De Santillán, Fernando. Declaración de lo que el Licenciado Fernando de Santillán, oidor de la Audiencia de Lima, proveyó para el buen gobierno, pacificación y defensa de Chile, 4 de junio de 1559. En: JARA, Álvaro y PINTO, Sonia. Fuentes para la Historia del trabajo en Chile. Santiago, Chile: Andrés Bello. 1982.

LARRAín, Guillermo. Estudio SAFP. Jubilación legal y anticipada.

PARdo, Adolfo. Historia de la mujer en Chile. La conquista de los derechos politicos en el S.XX. <En linea>. [Citado 6 de octubre de 2010]. Disponible en la World Wide Web: <http://www.critica.cl/html/pardo_01.html>

SÁNCHEZ, Karim. "El ingreso de la mujer chilena a la universidad y los cambios en la costumbre por medio de la ley 1872-1877”, en: Historia No 39, Vol. 2, Santiago, Chile, julio-diciembre 2006. Instituto de historia. Pontificia Universidad Católica de Chile.

Thayer Arteaga, William. Notas sobre génesis y desarrollo del derecho del trabajo en la República de Chile. En: Derecho del Trabajo: Normas y realidad, obra colectiva de homenaje al profesor don Ramón Luco, Santiago, Chile: editada por la Universidad Andrés Bello, 1993.

Todaro, Rosalba y Lerda, Sandra. "Estudio de Costos Laborales por Sexo", Informe Final de Consultoría al Servicio Nacional de la Mujer- SERNAM, Santiago, Chile, junio, 1996.

VAldés, Ximena, et al. Puertas adentro. Masculino y femenino en la familia contemporánea. Santiago, Chile: LOM, 2006.

Dirección del trabajo. Derechos de la mujer trabajadora. <En línea>. [Citado 6 de julio de 2010]. Disponible en la World Wide Web: <http://www.dt.gob.cl/legislacion/1611/w3-article-62965.html>

Historia de la INP. Integración ex cajas particulares. <En línea>. [Citado 8 de octubre de 2010]. Disponible en la World Wide Web: <http://portal.ips.gob.cl/portal/inp/datos_historia.htm>

Trabajo y equidad. Encuesta CASEN Diferencias salariales. <En línea>. [Citado 6 de octubre de 2010]. Disponible en la World Wide Web: <http://www.trabajoyequidad.cl/view/viewArticulos.asp?idArticulo=30>

Expectativa de vida <En línea>. [Citado 6 de Octubre de 2010]. Disponible en la World Wide Web: <http://web. latercera.cl/medio/articulo/0,0,3255_5726_35710797,00.html>

Conmemoración 90 años de la OIT. América en la OIT. <En línea>. [Citado 8 de octubre de 2010]. Disponible en la World Wide Web: <http://90.oit.org.pe/?p=7>

Rerum Novarum. Sobre la situación de los obreros. <En línea $>$. [Citado 9 de Octubre de 2010]. Disponible en la World Wide Web: <http://www.vatican.valholy_father/leo_xiiilencyclicals/documents/hf_l-xiii_enc_15051891_ rerum-novarum_sp.htmls 